\title{
Dinâmica espaço-temporal da dengue no Distrito Federal, Brasil: ocorrência e permanência de epidemias
}

\author{
Dengue spatiotemporal dynamics in the Federal District, Brazil: \\ occurrence and permanence of epidemics
}

Bruna Drumond (https://orcid.org/0000-0002-9007-8324) ${ }^{1}$

Jussara Ângelo (https://orcid.org/0000-0003-2324-1053) ${ }^{1}$

Diego Ricardo Xavier (http://orcid.org/0000-0001-5259-7732) ${ }^{2}$

Rafael Catão (https://orcid.org/0000-0003-2837-0364) ${ }^{3}$

Helen Gurgel(https://orcid.org/0000-0002-4250-6742) ${ }^{4}$

Christovam Barcellos (https://orcid.org/0000-0002-1161-2753) ${ }^{2}$

${ }^{1}$ Escola Nacional de Saúde Pública Sergio Arouca, Fiocruz. R. Leopoldo Bulhões 1480, Manguinhos. 21041-210 Rio de Janeiro RJ Brasil.bdrumonds@ gmail.com

${ }^{2}$ Instituto de Comunicação e Informação Científica e Tecnológica em Saúde, Fiocruz. Rio de Janeiro RJ Brasil.

${ }^{3}$ Departamento de Geografia. Universidade Federal do Espírito Santo.

Vitória ES Brasil.

${ }^{4}$ Departamento de

Geografia, Universidade de Brasília. Brasília DF Brasil.

\begin{abstract}
The specific characteristics of the Federal District (DF) favor the introduction, reproduction, dissemination, and permanence of dengue vector and viruses. Here, we aimed to analyze the spatiotemporal patterns of dengue epidemics in the Administrative Regions (RAs) of the DF from January 2007 to December 2017. We used Fourier partial series model to obtain a seasonal signature of the time series, which allowed calculating indicators of permanence (number of epidemic years, number of epidemic months per year, the proportion of epidemic months for the period) and time/ moment of epidemics (month of epidemic peak). A total of 82 epidemics were recorded in this peri$o d$. The RAs with the largest number of epidemic years were Varjão (5 epidemics), Gama, Lago Sul, and Sobradinho (4 epidemics). These last three RAs also had the highest proportions of epidemic months of the entire study period (9 epidemic months). The RAs with urban centrality function had an earlier epidemic peak than the others, in February and March. Epidemics showed high permanence values in RAs with different types of occupations, emphasizing the need to consider the social organization of space processes in dengue distribution studies.
\end{abstract}

Key words Dengue, Spatiotemporal analysis, Urban areas, Epidemics
Resumo O Distrito Federal (DF) apresenta características particulares que favorecem a introdução, reprodução, disseminação e permanência do vetor e dos vírus da dengue. O objetivo deste trabalho foi analisar os padrões espaço-temporais das epidemias de dengue nas Regiões Administrativas (RAs) do DF no período de janeiro de 2007 a dezembro de 2017. Utilizando o modelo da série parcial de Fourier, obteve-se uma assinatura sazonal da série temporal, o que permitiu calcular indicadores de permanência (número de anos epidêmicos, número de meses epidêmicos por ano, proporção de meses epidêmicos do período) e tempo/momento das epidemias (mês do pico epidêmico). Contabilizou-se um total de 82 epidemias nesse período. As RAs com maior número de anos epidêmicos foram Varjão (5 epidemias), Gama, Lago Sul e Sobradinho (4 epidemias). Essas últimas três RAs também tiveram as maiores proporções de meses epidêmicos de todo o período de estudo (nove). Verificou-se que as RAs com função de centralidade urbana possuem pico epidêmico mais cedo que as demais, nos meses de fevereiro e março. As epidemias apresentaram altos valores de permanência em RAs com diferentes tipos de ocupação, ressaltando-se a necessidade de considerar os processos de organização social do espaço nos estudos da distribuição da dengue.

Palavras-chave Dengue, Análise espaço-temporal, Áreas urbanas, Epidemias 


\section{Introdução}

A dengue ainda é um importante problema de Saúde Pública. O predomínio do ciclo de transmissão dessa endemia nas cidades remete a questões complexas relacionadas a processos como a urbanização, a mobilidade populacional e a globalização ${ }^{1,2}$. Presente nas áreas tropicais e subtropicais do globo, estima-se que 390 milhões de pessoas sejam infectadas pelos quatro sorotipos da dengue todo ano. Destas, apenas 96 milhões são sintomáticas ${ }^{3}$.

Em 2016, houve grandes epidemias em todo o mundo. Na região da Américas, foram reportados mais de 2,38 milhões de casos, cerca de 1,5 milhões de casos apenas no Brasil ${ }^{4}$. A dengue reemergiu no país na década de 1980 e desde então difundiu-se por quase todo o país, que tem passado por inúmeras epidemias. Atualmente, os quatro sorotipos da doença circulam no país, estabelecendo um cenário de hiperendemicidade em diversas cidades ${ }^{5,6}$.

Existe um número crescente de estudos sobre a distribuição espaço-temporal da dengue em cidades brasileiras e sua relação com condições socioeconômicas e ambientais ${ }^{7-11}$. A heterogeneidade espacial das condições de vida costuma ser um fator importante para explicar a distribuição dessa endemia ${ }^{7,9,11}$. Fatores relacionados às condições de saneamento, presença de favelas, renda, proporção de crianças e idosos, imunidade da população, densidade demográfica e intradomiciliar também estão presentes nesses estudos pois configuram-se como condições essenciais para a reprodução e a manutenção do vetor e do vírus ${ }^{2,7-11}$.

San Pedro et al. ${ }^{8}$ encontraram altas incidências de dengue em áreas de baixa, média e alta renda com diferentes práticas de estocagem de água na Região Oceânica de Niterói, ressaltando a necessidade de considerar os processos de organização do espaço que favorecem a produção da endemia. Xavier et al. ${ }^{12}$ contabilizaram 495 picos epidêmicos de dengue no período de 2000 a 2013 nos bairros do Rio de Janeiro. Esses autores identificaram os bairros com maior permanência de epidemias e discutiram as características e os processos de organização do espaço urbano que permitiram explicar o processo de difusão da doença.

A introdução de dengue em Brasília é relativamente recente, apesar do seu papel de centralidade, como capital do Brasil. Os primeiros casos autóctones de dengue no Distrito Federal (DF) ocorreram somente em 1997 e a primeira epidemia foi registrada em 2002, com 2.200 casos $^{13}$.
Atualmente, no DF, a dengue mantém-se endêmica, com registros de casos em todos os meses do ano ${ }^{14}$, apesar da sua altitude acima dos 1.000 metros e do seu inverno seco.

Brasília possui um dos maiores e principais aeroportos do país com um grande fluxo de pessoas do Brasil e do mundo. Além disso, a cidade possui elevados número populacional e densidade demográfica. Cabe destacar também que, nos últimos anos, a cidade tem passado por racionamento e problemas de abastecimento de água ${ }^{15}$. Todo esse contexto favorece a receptividade desse espaço urbano para a introdução, reprodução e permanência do vetor e dos vírus da dengue.

Sabe-se que a distribuição espacial das taxas de incidência e dos casos de dengue no DF não ocorre de maneira homogênea ${ }^{13,14}$. No entanto, ainda há um número pequeno de estudos feitos nesse espaço urbano ${ }^{13,14,16,17}$. A Capital Federal apresenta um espaço urbano polinucleado e fragmentado ${ }^{18-20}$, com diferentes padrões de uso do solo, renda e condições de saneamento. Além disso, seu processo de urbanização é marcado por uma ocupação irregular do solo, seja de baixa, média ou alta renda, em condomínios localizados em áreas vulneráveis do ponto de vista ambiental, hídrico ou de preservação do patrimônio histórico $^{18}$.

Diante desse contexto, o objetivo deste trabalho é analisar os padrões espaço-temporais das epidemias de dengue no DF no período de 2007 a 2017. Buscou-se evidenciar a temporalidade das epidemias por meio de indicadores de permanência e de tempo/momento de ocorrência.

\section{Métodos}

Este trabalho constitui-se em um estudo ecológico de abordagem espaço-temporal. Foram analisadas séries de casos de dengue no Distrito Federal (DF) e nas suas 31 Regiões Administrativas (RAs) no período de janeiro de 2007 a dezembro de 2017 para identificar epidemias e padrões temporais e espaciais da transmissão dessa endemia.

O DF está localizado entre os paralelos $15^{\circ} 30^{\prime}$ e $16^{\circ} 03^{\prime}$ de latitude sul e os meridianos de $47^{\circ} 25^{\prime}$ e $48^{\circ} 12^{\prime}$ de longitude oeste, na Região Centro-Oeste do Brasil. Possui uma área de $5.783 \mathrm{~km}^{2}$ e limita-se com municípios goianos e mineiros. Sua população, em 2018, estava estimada em 2.974.703 habitantes ${ }^{21}$.

Localizado no Planalto Central, o relevo do DF é caracterizado pelo predomínio de chapadas com altitudes que variam entre $950 \mathrm{~m}$ e $1.400 \mathrm{~m}^{22}$. 
O clima do DF é marcado por forte sazonalidade, com estação seca no inverno e úmida no verão. $\mathrm{Na}$ estação seca, de meados de maio até o início de setembro, há uma grande amplitude térmica diária, com dias mais quentes e noites frias, e baixa umidade relativa do $\mathrm{ar}^{23}$. A temperatura média anual é de $22^{\circ} \mathrm{C}$ e a precipitação anual acumulada é de $1.450 \mathrm{~mm}^{24}$.

Brasília possui características particulares por ter sido uma cidade criada para ser a capital do país e por possuir um traçado urbano com especificações de uso do solo. Apesar disso, não deixa de ter desigualdades semelhantes a outras cidades brasileiras, como a segregação espacial, produto de um processo de urbanização desigual ${ }^{19}$.

O DF possui um único município, Brasília. Esse território está organizado em 31 RAs - uma delas também com o nome de Brasília (Plano Piloto). A RA é a menor unidade utilizada por órgãos de planejamento, também usada neste trabalho como unidade espacial de análise

Foram utilizados dados referentes aos casos de dengue oriundos do Sistema de Informações de Agravos de Notificação (SINAN) notificados entre $1^{\circ}$ de janeiro de 2007 e 31 de dezembro de 2017 e disponibilizados pelo Ministério da Saúde (MS). Foram considerados para a análise os casos prováveis, confirmados por critério laboratorial ou clínico-epidemiológico, exceto os descartados por diagnóstico laboratorial negativo. Além dos dados descartados, foram retirados os casos considerados importados, os de residentes em outras UFs e aqueles sem informação de bairro ou outra forma de localização. Os dados foram agregados segundo o mês dos primeiros sintomas e RA de residência. As análises espaciais e o mapeamento dos indicadores foram feitos considerando a área ocupada das RAs, segundo dados obtidos no Geoportal da Secretaria de Estado de Desenvolvimento Urbano e Habitação (SEDUH) ${ }^{25}$.

Obteve-se uma assinatura sazonal da série temporal de casos de dengue para o DF e para cada uma das 31 RAs. Neste modelo, decompõese a série temporal em três senoides com ciclos sazonais de 12, 6 e 3 meses. Esses ciclos constituem uma série parcial de Fourier, uma abordagem semelhante a uma regressão periódica, mas que fornece parâmetros trigonométricos como amplitude e fase das senoides ao invés dos coeficientes de regressão das funções seno e cosseno. Essa abordagem permite o uso de parâmetros individuais das próprias harmônicas para quantificar o padrão temporal das epidemias ${ }^{12,26}$.

A função periódica anual de sazonalidade é dada pela fórmula a seguir:

$$
\begin{gathered}
Y_{t}=\alpha_{0}+\alpha_{1} t+\alpha_{2} t^{2}+Y_{1} \cos (2 \pi t / 12) \\
+\delta_{1} \sin (2 \pi t / 12)+Y_{2} \cos (2 \pi t / 6)+\delta_{2} \sin (2 \pi t / 6) \\
+Y_{3} \cos (2 \pi t / 3)+\delta_{3} \sin (2 \pi t / 3)+\varepsilon_{(t)}
\end{gathered}
$$

em que $\mathrm{Y}_{\mathrm{t}}$ é o número de casos de dengue no DF ou na RA no tempo $t, \alpha_{0}$ a média do número de casos no DF ou por RA, $\alpha_{1} t$ é a tendência linear e $\alpha_{2} \mathrm{t}^{2}$ corresponde à função matemática para representar a parábola de tendência, Y e $\delta$ são os coeficientes de regressão dos componentes periódicos (anual, semestral e trimestral) e $\varepsilon_{(\mathrm{t})}$ é o termo de erro com distribuição normal ${ }^{12,26,27}$.

Para caracterizar os padrões temporais de ocorrência de epidemias de dengue nas RAs, foram consideradas duas dimensões: a permanência das epidemias e o mês em que ocorre o pico epidêmico ${ }^{28,29}$. A "permanência" das epidemias foi medida por três indicadores: número de anos com epidemia, número de meses epidêmicos por ano e proporção de meses epidêmicos do período de estudo (2007 - 2017). O primeiro indicador permite verificar a persistência das epidemias ao longo dos anos, o segundo permite verificar a duração intra-anual das mesmas e o terceiro indica quanto tempo (em meses) do período foi considerado epidêmico.

Como indicador de tempo (momento) utilizou-se o mês do pico primário da assinatura sazonal da série temporal. Esse indicador refere-se ao mês em que ocorre a intensidade máxima da doença ${ }^{26}$.

Define-se como epidemia os períodos nos quais o número absoluto de casos supera o limiar de casos esperados, considerando a série histórica suavizada de casos a partir do comportamento sazonal e das alterações anuais além do componente aleatório que configura situação epidêmica. As observações dentro do intervalo de confiança de 95\% são consideradas como variabilidade irregular. As observações que ultrapassarem esse intervalo são consideradas como período epidêmico ou anomalias ${ }^{12,26}$.

Os resultados obtidos não se referem, portanto, a taxas de incidência, mas a indicadores de temporalidades. $\mathrm{O}$ uso do número absoluto de casos torna-se adequado para este estudo visto que o intuito é considerar a evolução do número de casos dentro de uma mesma RA e, a partir disso, identificar a ocorrência e a permanência de epidemias.

O modelo da assinatura sazonal e os parâmetros sazonais da série foram obtidos por meio do software EPIPOI ${ }^{26}$. Todos os indicadores foram organizados em bancos de dados geográficos e apresentados em mapas temáticos construídos 
no software de Sistema de Informações Geográficas (SIG) ArcGIS 10.4 .

Esta pesquisa foi aprovada pelo Comitê de Ética em Pesquisa (CEP) da Escola Nacional de Saúde Pública (ENSP) da Fundação Oswaldo Cruz (Fiocruz).

\section{Resultados}

No período de 1 de janeiro de 2007 a 31 de dezembro de 2017 foram registrados 70.495 casos prováveis de dengue no Distrito Federal. Destes, aproximadamente $74 \%$ ocorreram nos anos de 2010, 2013, 2014 e 2016, considerados anos epidêmicos.

O Gráfico 1 mostra um alto número de casos no ano de 2015, com pico em maio, porém não ultrapassando o limite superior para epidemias. Considerando a sazonalidade da série temporal, a epidemia de 2010 teve início em março, antes do pico sazonal calculado para toda a série histórica pelo modelo, em abril. Em 2013, o pico epidêmico ocorreu junto com o pico da assinatura sazonal da série. Em 2014, ocorreu um mês depois, em maio. Já em 2016, a epidemia antecipou-se de forma expressiva, começando em janeiro e tendo seu pico em fevereiro.

No período de estudo analisado, verificou-se um aumento expressivo no número de casos a partir de 2010. Em geral, o número de casos foi maior nos primeiros semestres, particularmente entre o final do verão e o início do outono, perío- do que coincide com o final do período chuvoso. O mês com o maior número de casos do período foi fevereiro de 2016, ano em que houve uma antecipação do período epidêmico (Gráfico 1).

A partir da análise das assinaturas sazonais por RA, contabilizou-se 82 epidemias entre 2007 e 2017. A RA Varjão apresentou comportamento epidêmico em 5 anos. Gama, Lago Sul e Sobradinho registraram epidemias durante 4 anos. Catorze RAs (45\%) apresentaram 3 epidemias, dez RAs registraram 2 epidemias e apenas Estrutural, Guará e Plano Piloto tiveram uma única epidemia no período (Figura 1).

O mapa da Figura 2 mostra o mês de pico primário anual da assinatura sazonal da série temporal de cada RA. Plano Piloto, Brazlândia e Taguatinga tiveram pico em fevereiro. Vicente Pires, Guará e Sobradinho, em março. O mês de maior frequência foi abril, com 23 (74\%) das RAs. Varjão, Sudoeste/Octogonal e Itapoã foram as RAs que apresentaram pico mais tardio, em maio.

Enquanto o mapa do número de epidemias (Figura 1) mostra a persistência das epidemias ao longo dos anos, os mapas da Figura 3 mostram a persistência intra-anual das epidemias. Observase que no ano de 2010 as epidemias ocorreram de maneira dispersa, por muitas RAs, e tiveram pouca duração, de maneira geral. Nesse ano, 23 (74\%) das RAs tiveram apenas 1 mês epidêmico. Nas RAs Candangolândia, Plano Piloto, Recanto das Emas e Vicente Pires a epidemia durou 2 meses. Apenas em Planaltina houve uma persistência da epidemia por 3 meses.

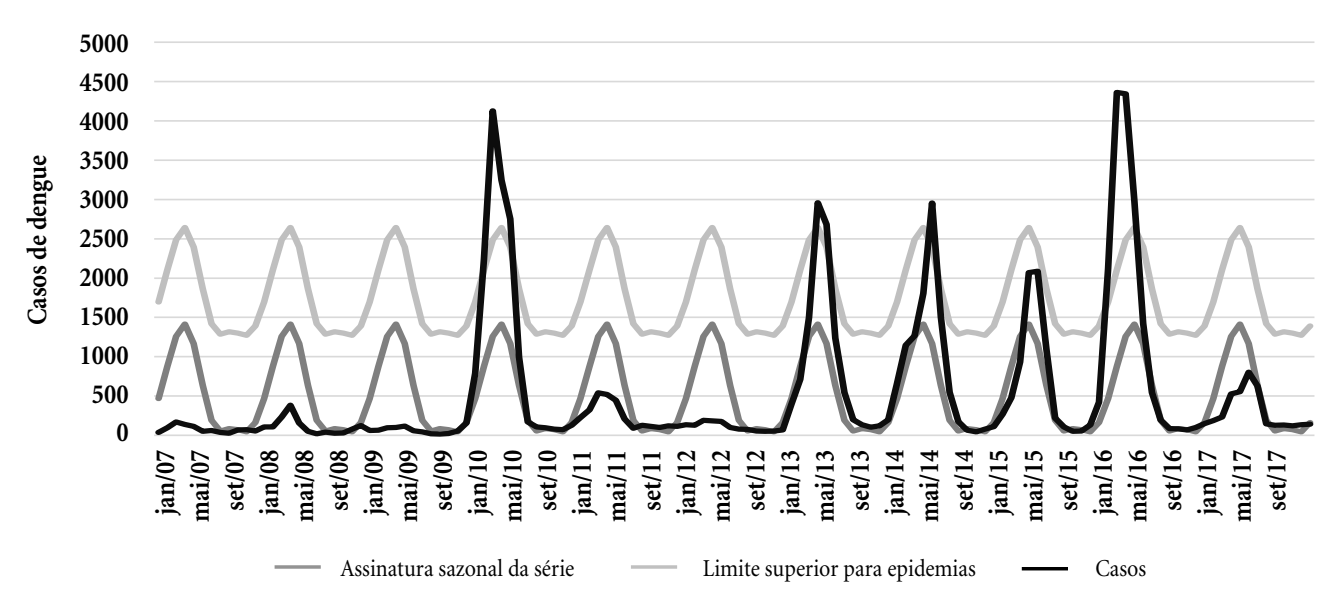

Gráfico 1. Série temporal de casos de dengue no Distrito Federal, Brasil, assinatura sazonal e limite superior para epidemias no período de 1 de janeiro de 2007 a 31 de dezembro de 2017. 


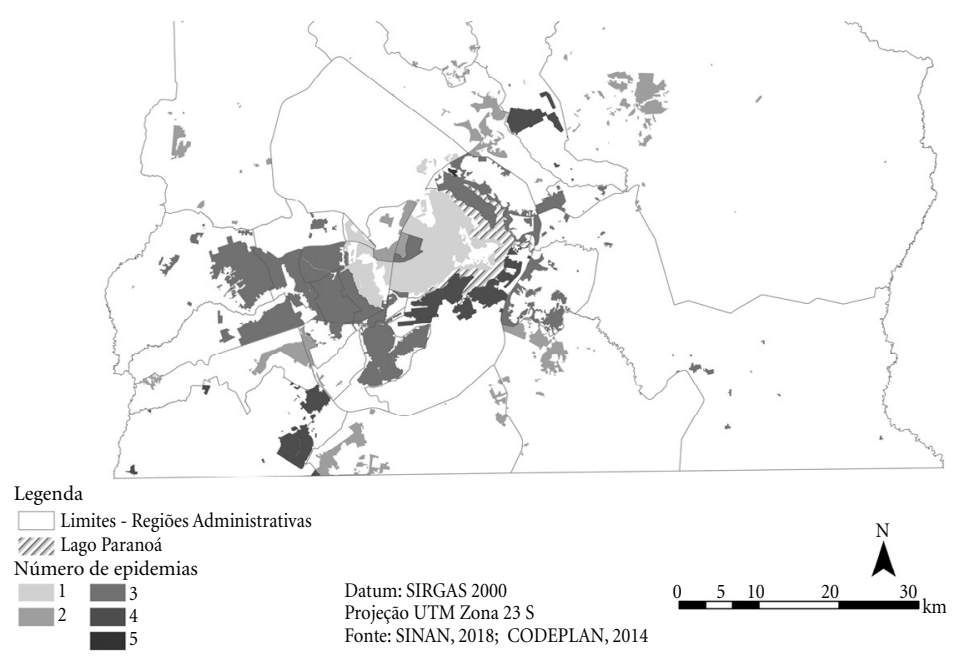

Figura 1. Distribuição espacial do número de epidemias por Região Administrativa do Distrito Federal (2007-2017).

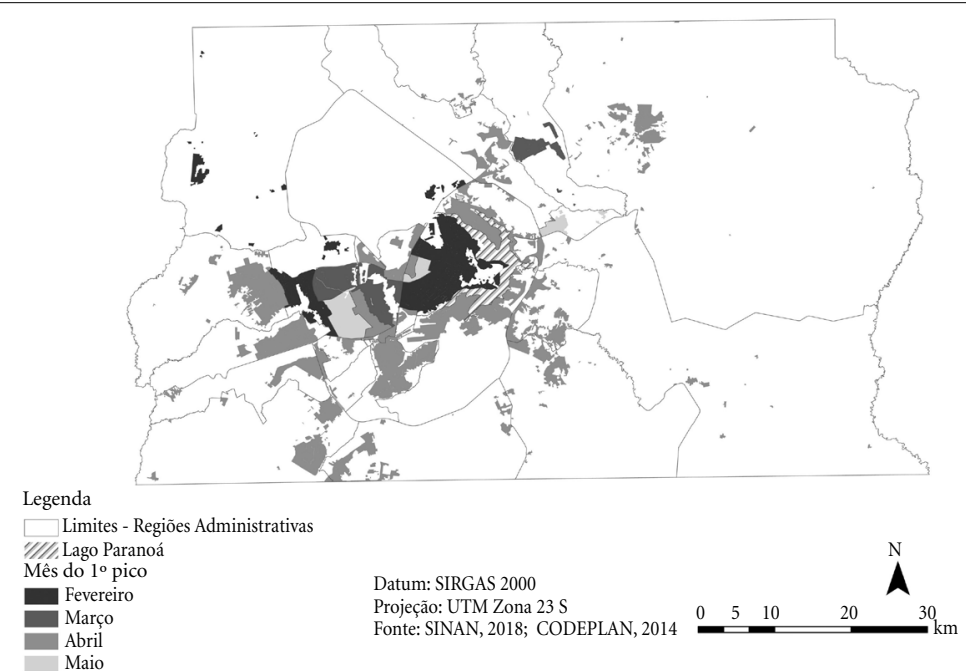

Figura 2. Mês do pico primário anual das assinaturas sazonais das séries temporais de dengue das RAs do DF.

Em 2013, Brazlândia, Lago Norte, Park Way e Varjão registraram apenas 1 mês com comportamento epidêmico. Nas RAs Lago Sul, Riacho Fundo I, Samambaia, Sudoeste/Octogonal e Taguatinga a epidemia durou 2 meses. Em Águas Claras e Ceilândia durou 3 meses. Somente em Vicente Pires houve uma persistência da epidemia por 4 meses seguidos.

Em 2014, Candangolândia, Itapoã, Jardim Botânico, Núcleo Bandeirante e Paranoá tiveram apenas 1 mês com comportamento epidêmico. Santa Maria, Sobradinho e Sobradinho II tiveram dois meses de epidemia. Varjão registrou 3 meses epidêmicos e Fercal e Gama tiveram uma epidemia com duração de 4 meses.

$\mathrm{O}$ ano de 2015 não foi considerado um ano epidêmico pela assinatura sazonal da série de casos de dengue do DF como um todo. Entretanto, ao se aplicar o modelo para as RAs, verifica-se que 7 (22\%) delas apresentaram comportamento 

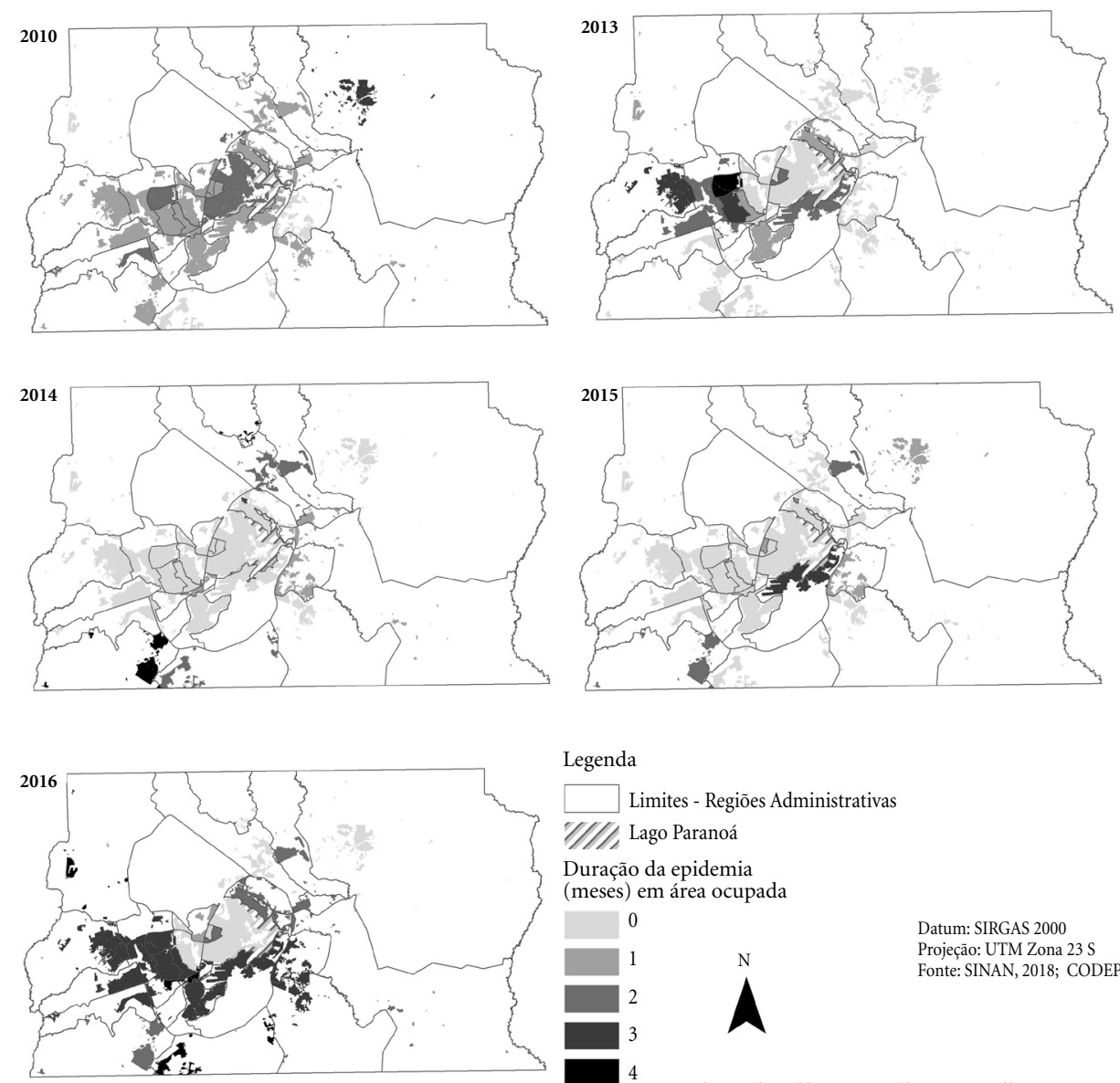

Legenda

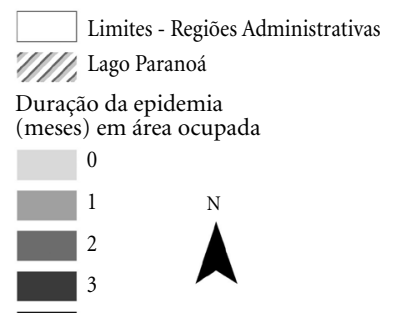

Figura 3. Distribuição espacial do número de meses epidêmicos para dengue por Região Administrativa do Distrito Federal.

epidêmico. Cruzeiro, Planaltina, Jardim Botânico e Varjão registraram apenas 1 mês de epidemia. Sobradinho e Gama tiveram 2 meses de comportamento epidêmico e no Lago Sul a epidemia durou 3 meses.

Em 2016, as RAs Setor de Indústria e Abastecimento (SIA) e Varjão registraram 1 mês com comportamento epidêmico. Lago Norte, Sudoeste/Octogonal, Itapoã, Paranoá, Sobradinho e Gama tiveram 2 meses de epidemia. Doze (39\%) RAs tiveram 3 meses epidêmicos e em Brazlândia, Riacho Fundo I, Núcleo Bandeirante e Santa Maria a epidemia persistiu por 4 meses.

O mapa da proporção de meses epidêmicos do período de estudo (2007 - 2017) (Figura 4) mostra que as epidemias foram mais persistentes nas RAs Gama, Lago Sul e Vicente Pires, com 9
(6,82\%) meses epidêmicos. Dez (32\%) RAs tiveram entre 6 e $7(3,80 \%$ - 5,30\%) meses epidêmicos. Treze (42\%) RAs tiveram entre 3 e 5 ( $1,53 \%$ - 3,79\%); Cruzeiro, Plano Piloto e SIA tiveram 2 $(1,52 \%)$ meses e Estrutural e Guará apresentaram apenas 1 mês epidêmico.

\section{Discussão}

No Distrito Federal (DF), a dengue mantém-se hiperendêmica, com os quatro sorotipos circulando e com casos registrados durante todos os meses do ano, cenário semelhante às grandes cidades brasileiras. A existência de muitos sorotipos circulando em uma grande população é um dos principais fatores da ocorrência contínua de 
Figura 4. Distribuição espacial da proporção de meses epidêmicos (dengue) de todo o período por RA do DF.

epidemias ao longo dos $\operatorname{anos}^{28}$. No período de estudo, foram observadas quatro epidemias de dengue no DF: em 2010, 2013, 2014 e 2016.

Na epidemia de 2010, os sorotipos circulantes foram o DENV-1, o DENV-2 e o DENV-3. Na epidemia de 2013 eram os sorotipos DENV-1 e DENV-4. No ano seguinte, apenas o DENV-1 foi detectado. Já na epidemia de 2016, os quatros sorotipos circulavam no DF, caracterizando um cenário hiperendêmico. Em todas as epidemias, no entanto, houve predomínio do DENV-128-31.

A análise da sazonalidade da dengue no DF permitiu observar que, em geral, nos anos em que as epidemias se iniciam antes, como ocorreu em 2010, em fevereiro, e em 2016, em janeiro, a intensidade do pico epidêmico (considerando o valor do mês com o maior número de casos) e a duração da epidemia é maior que nos anos de epidemias mais tardias. Supõe-se que as epidemias que se iniciam mais tarde não permitem a manutenção de um elevado número de casos devido às mudanças de temperatura e precipitação a partir de maio, variáveis essenciais para a sobrevivência do vetor.

Por outro lado, as epidemias que ocorrem mais tardiamente podem apresentar uma recorrência no ano seguinte, pois podem não atingir um número suficiente de suscetíveis para que a população fique imune. Isso pode ter ocorrido nas epidemias de 2013 e 2014.
Nos anos em que as epidemias começaram mais cedo, 2010 e 2016, também houve uma maior capacidade de espalhamento das epidemias, que ocorreram em quase todas as RAs. Resultados semelhantes foram encontrados por Xavier et al. ${ }^{12}$ ao analisarem as epidemias de dengue na cidade do Rio de Janeiro.

Os indicadores temporais permitiram compreender a persistência das epidemias considerando os anos e os meses de ocorrência. As RAs Varjão e Lago Sul, com 5 e 4 anos epidêmicos no período de estudo, respectivamente, estão localizadas na Zona de Uso Controlado I do Plano Diretor de Ordenamento do Território (PDOT) vigente no DF. Essa área faz parte da bacia do Lago Paranoá e possui uma paisagem urbana marcada por espaços livres públicos e privados. No PDOT destaca-se o papel dessa Zona no que tange a preservação do conjunto tombado e da paisagem visual do Plano Piloto ${ }^{31}$.

Essa área é considerada de grande importância ambiental, com presença de áreas protegidas e de interesse ambiental. O uso é predominantemente habitacional de baixa densidade, favorecendo a manutenção das áreas verdes. No entanto, a RA Varjão é considerada um enclave de alta densidade nessa Zona. O perfil socioeconômico da população da Zona Urbana de Uso Controlado I é caracterizado por média-alta e alta renda nas áreas de menor densidade demográfica, 
como o Lago Sul, e de baixa renda nas áreas de maior densidade, como o Varjão ${ }^{31}$.

Cabe destacar que essas duas RAs não são populosas. A RA Lago Sul ocupa a 24a posição em tamanho de população, estimada em 28.981 no ano de 2015. Já, a RA Varjão apresenta a terceira menor população, com apenas 8.453 habitantes $^{32}$. Uma população pequena pode implicar num número pequeno de suscetíveis. Isso explicaria o fato dessa RA apresentar recorrência anual de epidemias, mas curta duração em meses. Essa RA apresentou apenas um mês epidêmico em 4 dos anos em que apresentou epidemia. A exceção foi o ano de 2013, em que a epidemia durou 3 meses.

As RAs Sobradinho e Gama estão localizadas na Zona Urbana Consolidada. São áreas servidas de infraestrutura e equipamentos urbanos. Possuem média e baixa densidade populacional com alguns enclaves de alta densidade. Sobradinho, no entanto, é considerada uma localidade com limitações de abastecimento de água e esgotamento sanitário pelo PDOT de $2009^{31}$, o que pode favorecer a transmissão e a manutenção da dengue. A possível escassez ou interrupção do serviço de abastecimento de água faz com que as famílias tenham a necessidade de armazenar água em toneis e outros recipientes para consumo, favoráveis à reprodução do vetor ${ }^{8,33,34}$.

A RA Gama foi uma das primeiras a registrar transmissão autóctone da dengue no DF, em $1997^{35}$. Essa RA tem uma população estimada em 134.111 habitantes, com uma renda domiciliar média mensal estimada em 5,76 salários mínimos (SM), a $17^{\text {a }}$ posição entre as $31 \mathrm{RAs}^{32}$.

Nota-se, portanto, que as áreas urbanas em que as epidemias têm sido mais recorrentes possuem diferentes padrões de ocupação. San Pedro et al. $^{8}$ analisaram localidades com incidências semelhantes de dengue, mas com padrões urbanísticos e socioeconômicos diferentes na Região Oceânica de Niterói. Esses autores identificaram fatores de risco distintos determinando a transmissão da dengue. $\mathrm{O}$ primeiro referente à limitação da oferta de serviço de abastecimento de água e escassez de recursos financeiros. O segundo relacionado a uma abundância de recursos, que permite práticas de estocagem de água ${ }^{8}$.

Nas áreas com esse segundo tipo de risco foi identificado um grande número de piscinas ${ }^{8}$, assim como ocorre na RA Lago Sul. Esse resultado, no entanto, deve ser visto com ressalva, pois recipientes de até $100 \mathrm{ml}$ e acima de 5.000 litros podem ser considerados pouco produtivos quanto à capacidade de produzir formas adultas do vetor ${ }^{34}$.

Além da permanência das epidemias ao longo do período de estudo, também foi analisada a duração intra-anual das epidemias. Em 2016, assim como em 2010, a epidemia atingiu quase todas as RAs, com algumas exceções, e nesses dois anos a epidemia teve uma longa duração no DF.

Entretanto, em 2010, verifica-se uma curta duração das epidemias nas RAs, em geral. Essa dinâmica sugere um possível deslocamento das epidemias pelo espaço urbano. Em 2016, ao contrário, as epidemias persistiram por mais tempo também nas RAs. Isso pode estar relacionado ao fato de, em 2016, os 4 sorotipos da dengue estarem circulando, além de já circularem também os vírus da chikungunya e da zika ${ }^{36}$. É possível que alguns casos de zika ou chikungunya tenham sido notificados como dengue por critério clínico-epidemiológico e vice-versa.

Em 2013 e 2014, a duração das epidemias foi de apenas 1 mês para o DF. No entanto, algumas RAs apresentaram epidemia de longa duração nesses anos. As RAs Vicente Pires, em 2013, e Gama, em 2014, registraram epidemias com 4 meses de duração. Isso pode indicar condições favoráveis para a reprodução do vetor e, consequentemente, para a transmissão e permanência da doença nessas RAs.

Essas mesmas RAs, Vicente Pires e Gama, e a RA Lago Sul apresentaram a maior proporção de meses epidêmicos do período. Vicente Pires é considerada pelo PDOT vigente como Zona Urbana de Qualificação e Expansão. No PDOT anterior, de 1997, essa área era destinada ao uso rural, mas sofreu um intenso processo de ocupação urbana. Nessa área verificam-se renda e densidade populacional médias ${ }^{31}$.

A ocupação dessa RA se deu a partir de assentamentos informais e parcelamento ilegal do solo. Boa parte de sua área está localizada em Áreas de Preservação Permanente (APP) e Ambiental (APA), com muitas nascentes que foram soterradas para edificações ${ }^{18,37}$.

Ela configura-se como cidade-dormitório, com apenas $13,75 \%$ de seus moradores trabalhando na própria RA. É uma área predominantemente residencial com habitações unifamiliares e com presença de alguns condomínios horizontais. A baixa oferta de empregos e serviços implica em uma grande dependência do Plano Piloto e configura-se como herança da urbanização modernista $^{38}$.

Cabe destacar, no entanto, que a RA Vicente Pires possui renda média domiciliar de 10,92 SM, oitava maior renda do $\mathrm{DF}^{32}$. Essa RA encontra-se em um dos principais eixos rodoviários, a Estrada Parque Taguatinga (EPTG), que concentra as RAs mais populosas ${ }^{38}$. É nesse eixo que ocorre um prin- 
cípio de conurbação, rompendo com o padrão polinucleado de ocupação do solo urbano ${ }^{18,20}$.

As RAs Gama, Lago Sul e Vicente Pires também possuem alta densidade domiciliar (número de pessoas por domicílio) ${ }^{14}$ e, possivelmente, disponibilidade de criadouros do vetor. Outro aspecto em comum entre essas RAs é a predominância do domicílio do tipo casa (ou habitação unifamiliar). Sabe-se que a habitação do tipo apartamento pode ser um fator de proteção às epidemias de arboviroses como a dengue, pois recipientes que se configuram como criadouros potenciais para o vetor são mais presentes em casas e seus quintais do que em apartamentos ${ }^{39}$.

A difusão da dengue no espaço intraurbano do DF não foi o foco deste trabalho. Entretanto, a análise do indicador de tempo (momento), apontando o mês de ocorrência dos picos primários das assinaturas sazonais das séries temporais de dengue das RAs sugere uma difusão das epidemias a partir das RAs Plano Piloto, Brazlândia e Taguatinga, com pico primário ocorrendo em fevereiro.

O Plano Piloto integra a Zona Urbana do Conjunto Tombado. Essa Zona corresponde à poligonal tombada pelo Instituto do Patrimônio Histórico e Artístico Nacional (IPHAN) e reconhecido pela Organização das Nações Unidas para a Educação, a Ciência e a Cultura (UNESCO) como Patrimônio Cultural da Humanidade. Assim, a ocupação dessa Zona segue normas e restrições de uso do solo com vistas à preservação do patrimônio tombado ${ }^{31,40}$.

Essa Zona configura-se como centralidade principal do espaço intraurbano do DF. Ela centraliza atividades administrativas do DF e do Governo Federal, concentrando a maior oferta de emprego, bem como serviços e equipamentos de alcance regional. Assim, esta Zona tem uma demanda intensa por novos espaços para essas atividades e por infraestrutura urbana de transporte $^{31}$. Nessa RA há o predomínio de população média-alta e alta renda. A densidade demográfica é considerada média e há o predomínio da habitação do tipo apartamento ${ }^{14,31,32}$.

A RA Taguatinga foi a primeira cidade-satélite, criada ainda em 1958, antes da inauguração do Plano Piloto e localizada a $25 \mathrm{~km}$ deste. Foi construída para abrigar a população de migrantes e operários que já habitavam o canteiro de obras da capital em acampamentos, barracos e favelas. Essa população foi transferida para Taguatinga, onde cada família ganhou um lote, porém sem infraestrutura urbana ${ }^{41}$.

Ao longo dos anos, Taguatinga se estruturou como a mais completa das "cidades-satélites"
É bem servida de infraestrutura e equipamentos urbanos e está na Zona Urbana Consolidada do PDOT $^{31}$. Essa RA pode ser considerada um subcentro no $\mathrm{DF}^{42}$, possuindo o segundo maior número de postos de trabalho do DF. No entanto, cabe destacar que esse percentual é de apenas $7,71 \%$, enquanto o Plano Piloto detém 41,53\% dos postos do trabalho do $\mathrm{DF}^{32}$.

A RA Brazlândia é um núcleo urbano com origem na década de 1930, antecedendo a implantação da Capital. Passou por um processo de expansão com princípios de urbanismo modernista que pouco se conecta ao espaço urbano "pré-moderno", o que implica num tecido urbano fragmentado, com diversas configurações ${ }^{20}$.

É considerado um núcleo urbano isolado no $\mathrm{DF}^{20}$. Depois do Plano Piloto (89,35\%), é a segunda RA com maior percentual de pessoas que trabalham na mesma RA que residem $(53,49 \%)^{32}$, o que expressa uma independência em relação a centralidade principal do espaço urbano do DF, localizada a cerca de $52 \mathrm{~km}$ de distância. Os núcleos urbanos mais próximos de Brazlândia, no DF, são Taguatinga e Ceilândia, há cerca de 30 km e o município goiano de Águas Lindas de Goiás, há cerca de $18 \mathrm{~km}^{38}$

A RA Brazlândia está inserida na Zona Urbana de Uso Controlado II. Possui uma população estimada em 51.816 habitantes e uma baixa densidade demográfica. É considerada uma das maiores produtoras de hortifrutigranjeiros do DF, porém a maior parte de sua população ocupada concentra-se no setor terciário. A renda da população é considerada baixa (apenas 1,25 SM per capita) ${ }^{31,32}$.

Assim, das três RAs com pico primário do modelo em fevereiro, duas configuram-se como centralidades. Das três que tiveram pico em março, Guará e Sobradinho também constituem-se em centralidades e Vicente Pires está no principal eixo de expansão urbana que liga Taguatinga ao Plano Piloto $^{38,42}$.

Ao analisar as epidemias de dengue, chikungunya e zika em Salvador nos anos de 2015 e 2016, Santana ${ }^{43}$ verificou que o principal foco de transmissão se dá em uma área que se estende do oeste do município à região central e que a difusão ocorre por expansão. No entanto, cabe destacar que o espaço intraurbano de Salvador, mais antigo e consolidado, é expressivamente diferente do espaço intraurbano polinucleado e modernista do DF.

Catão ${ }^{29}$, ao analisar a difusão do vetor e da dengue no estado de São Paulo, verificou que ambos apresentam difusão mista - hierárquica e por contágio. Esse autor verificou que as cidades 
e aglomerações urbanas e metropolitanas com maior centralidade e com tamanho populacional que permite a endemicidade da dengue em áreas mais propícias da perspectiva ambiental atuam como difusoras da dengue em suas regiões mais próximas e mesmo em todo o estado ${ }^{29}$.

A partir dos resultados do indicador de tempo (momento) encontrados para as RAs é possível afirmar que as centralidades do espaço intraurbano do DF - Plano Piloto, Taguatinga, Guará e Sobradinho ${ }^{38,42}$ - apresentam picos epidêmicos mais cedo (fevereiro e março) que as demais RAs.

As limitações deste trabalho referem-se aos dados da dengue, pois foram considerados casos prováveis por critério laboratorial e clínico-epidemiológico. Como os sintomas da dengue são muito semelhantes aos de outras arboviroses, como chikungunya e zika, pode ter havido um viés de notificação clínica-epidemiológica. Além disso, a dengue apresenta casos assintomáticos, existindo a possibilidade de subnotificação.

Outra limitação refere-se ao fato deste trabalho não ter levado em consideração indicadores socioambientais que refletem os determinantes da dengue. Por meio desses indicadores seria possível fazer análises mais robustas, identificando possíveis correlações, bem como caracterizando as áreas por meio de tipologias intraurbanas de vulnerabilidade do social e receptividade às arboviroses e ao vetor. Essa limitação torna-se, assim, a principal sugestão de desdobramento de pesquisas futuras

\section{Considerações finais}

Os principais achados deste trabalho dizem respeito à identificação dos padrões de transmissão da dengue no DF, levando em consideração a permanência de epidemias, sua duração e variação sazonal da arbovirose. Essas informações são muito relevantes para as atividades de vigilância em saúde e colaboram para ampliar a compreensão dos processos endêmicos-epidêmicos.

A partir desses indicadores, a vigilância pode se preparar com antecedência e de maneira mais eficaz, considerando as particularidades dos padrões de transmissão de cada área, bem como dos tipos de ocupação e de processos que nelas ocorreram. Destaca-se o uso de ferramentas de análise espacial, que configuram-se como instrumentos essenciais para essas atividades na identificação das áreas e na caracterização dos padrões de transmissão das arboviroses.

Os maiores valores de indicadores de permanência das epidemias ao longo dos anos e de duração intra-anual das epidemias foram localizados em áreas com diferentes tipos de ocupação do espaço e com diferentes perfis de renda e de densidade demográfica. Foram encontradas altas permanência e duração em áreas de renda baixa e alta densidade populacional; de renda média e diferentes densidades, porém com limitações de abastecimento de água; de renda alta, com piscinas e jardins. Assim, esses tipos de ocupação diferentes, mas com semelhantes indicadores de permanência e duração, sugerem que a dengue persiste por mais tempo em áreas com processos de produção do espaço urbano variados que propiciam diferentes práticas e necessidades de estocagem de água.

Outro achado importante diz respeito ao momento em que as epidemias se iniciam. Verificouse que as centralidades do espaço intraurbano no DF possuem picos epidêmicos mais cedo que a maioria das outras localidades. Essa informação pode ser relevante pois as atividades de vigilância das demais áreas podem se preparar diante da notificação do aumento de casos nas áreas centrais.

O método empregado neste artigo pode ser aplicado por serviços de vigilância de saúde em pequenas áreas, que não dispõe de estimativas populacionais para o cálculo de taxas de incidência. Utiliza um diagrama de controle, no entanto de forma mais robusta que o usual, porque considera simultaneamente o comportamento sazonal, alterações anuais, além do componente aleatório que configura situação epidêmica. Assim, o método empregado depende unicamente de dados de notificação de casos de dengue desagregados por bairros, distritos, ou área de cobertura do posto de saúde. Esses dados estão disponíveis em grande parte dos municípios brasileiros. A sua utilização como rotina dos serviços de vigilância em saúde permitiria a deteç̧ão de surtos localizados e sua tendência de difusão nas cidades. 


\section{Colaboradores}

B Drumond foi responsável pela concepção e desenvolvimento do estudo, pela interpretação e análise dos dados e pela redação do artigo. C Barcellos e J Ângelo participaram da concepção, orientação, revisão crítica da interpretação e análise dos dados e redação do artigo. DR Xavier participou da revisão crítica da interpretação e análise dos dados e do conteúdo intelectual e da redação do artigo. R Catão e H Gurgel colaboraram com revisão crítica da análise dos dados e do conteúdo intelectual e aprovaram a versão final do artigo.

\section{Agradecimentos}

À Coordenação de Aperfeiçoamento de Pessoal de Nível Superior (CAPES) pela bolsa de mestrado da primeira autora.

\section{Referências}

1. Czeresnia D, Ribeiro AM. O conceito de espaço em epidemiologia: uma interpretação histórica e epistemológica. Cad Saude Publica 2000; 16(3):595-605.

2. Gubler DJ. Dengue, Urbanization and Globalization: The Unholy Trinity of the 21(st) Century. Trop Med Health. 2011; 39(4 Supl.):3-11.

3. Bhatt S, Gething PW, Brady OJ, Messina JP, Farlow AW, Moyes CL, Drake JM, Brownstein JS, Hoen AG, Sankoh O, Myers MF, George DB, Jaenisch T, Wint GR, Simmons CP, Scott TW, Farrar JJ, Hay SI. The global distribution and burden of dengue. Nature 2013; 496(7446):504-507.

4. World Health Organization (WHO). Dengue and severe dengue. WHO Fact Sheet dengue and severe dengue. Geneva: WHO; 2020.

5. Catão R, Guimarães R. Mapeamento da reemergência do dengue no Brasil-1981/82-2008. Hygeia 2011; 7(13):173-185.

6. Brasil. Ministério da Saúde (MS). Dengue. Descrição da Doença. 2018. [acessado 2018 Maio 30]. Disponível em: http://portalms.saude.gov.br/saude-de-a-z/dengue/descricao-da-doenca

7. Flauzino RF, Souza-Santos R, Oliveira RM. Dengue, geoprocessamento e indicadores socioeconômicos e ambientais: um estudo de revisão. Rev Panam Salud Pública 2009; 25(5):456-461.

8. San Pedro A, Souza-Santos R, Sabroza PC, Oliveira RM. Condições particulares de produção e reprodução da dengue em nível local: estudo de Itaipu, Região Oceânica de Niterói, Rio de Janeiro, Brasil. Cad Saude Publica 2009; 25(9):1937-1946.

9. Costa Resendes AP, Silveira NAPR, Sabroza PC, Souza-Santos R. Determinação de áreas prioritárias para ações de controle da dengue. Rev Saude Publica 2010; 44(2):274-282.

10. Cordeiro R, Donalisio MR, Andrade VR, Mafra AC, Nucci LB, Brown JC, Stephan C. Spatial distribution of the risk of dengue fever in southeast Brazil, 20062007. BMC Public Health. 2011; 11:355.

11. Souza LS, Barata RDCB. Diferenciais intraurbanos na distribuição de dengue em Cuiabá, 2007 e 2008 Intra-urbandiferentials in dengue distribution, Cuiabá , 2007-2008. Rev Bras Epidemiol 2012; 15(4):761-770.

12. Xavier DR, Magalhães MAFM, Gracie R, Reis IC, Matos VP, Barcellos C. Difusão espaço-tempo do dengue no Município do Rio de Janeiro, Brasil, no período de 2000-2013. Cad Saude Publica 2017; 33(2):e00186615.

13. Catão RDC, Guimarães RF, Abílio O, Júnior DC, Trancoso RA. Análise Da Distribuição Do Dengue No Distrito Federal. Espaço e Geogr 2009; 12(1):81-103.

14. Silveira BD, Siqueira R, Gurgel H, Ramalho WM. Dengue e desigualdades socioespaciais no Distrito Federal, Brasil (2007-2014). Dourados: UFGD; 2017.

15. Distrito Federal. Resolução n ${ }^{\circ} 20$, de 7 de novembro de 2016. Declara o estado de restrição de uso dos recursos hídricos, estabelece o regime de racionamento do serviço de abastecimento de água nas localidades atendidas pelos reservatórios do Descoberto e Santa Maria e dá outras providências. Diário Oficial do Distrito Federal 2016; 7 nov.

16. Siqueira RV, Gurgel HC, Silveira BD, Ramalho WM. Relações entre a dinâmica ambiental e a dengue no Distrito Federal, Brasil. Rev Bras Geogr Médica e da Saúde - Hygeia 2017; 13(26):1980-1726. 
17. Gregório L. Relações entre a dinâmica espaço-temporal da dengue e os padrões urbanos no Distrito Federal, Brasil [tese]. Brasília: UnB; 2018.

18. Paviani A. Geografia Urbana do Distrito Federal: Evolução e Tendências. Espaço e Geogr 2007; 10(1):1-22.

19. Ferreira ICB. O processo de urbanização e a produção do espaço metropolitano de Brasília. In: Paviani A, organizador. Brasília, Ideologia e Realidade: Espaço Urbano Em Questão. Brasília: UnB; 2010. p. 61-81.

20. Holanda F, Medeiros V, Ribeiro R, Moura A. A configuração da Área Metropolitana de Brasília. In: Ribeiro R, Tenório G, Holanda F, organizadores. Brasília: Transformações Na Ordem Urbana. Rio de Janeiro: Letra Capital; 2015. p. 64-97.

21. Instituto Brasileiro de Geografia e Estatística (IBGE). @cidades. 2019. [acessado 2018 Maio 30]. Disponível em: https://cidades.ibge.gov.br/brasil/df/panorama

22. Distrito Federal. Atlas Do Distrito Federal 2017. Brasília; 2017. [acessado 2018 Maio 30]. Disponível em: http://www.codeplan.df.gov.br/wp-content/uploads/2018/05/Atlas-do-Distrito-Federal-2017.pdf.

23. Steinke ET, Steinke VA. Fatores determinantes do período de seca no Distrito Federal. Bol Gaúcho Geogr 2000; 26(1):244-254.

24. Instituto Nacional de Meteorologia do Brasil (INMET). Normal Climatológica do Brasil 1981-2010. [acessado 2018 Maio 30]. Disponível em: http://www. inmet.gov.br/portal/index.php? $r=$ clima/normaisClimatologicas

25. Distrito Federal S. Infraestrutura de Dados Espaciais - IDE/DF. [acessado 2018 Maio 30]. Disponível em: https://www.geoportal.seduh.df.gov.br/mapa/\#

26. Alonso WJ, McCormick BJJ. EPIPOI: A user-friendly analytical tool for the extraction and visualization of temporal parameters from epidemiological time series. BMC Public Health 2012; 12:982.

27. Schuck-paim C, Simonsen L, Miller MA, Moura FE, Fernandes RM, Carvalho ML, Alonso WJ. Were Equatorial Regions Less Affected by the 2009 Influenza Pandemic? The Brazilian Experience 2012; 7(8):e41918.

28. Barcellos C, Lowe R. Expansion of the dengue transmission area in Brazil: The role of climate and cities. Trop Med Int Heal 2014; 19(2):159-168.

29. Catão R. Expansão e Consolidação do Complexo Patogênico do Dengue no Estado de São Paulo: Difusão Espacial e Barreiras Geográficas [tese]. Presidente Prudente: UNESP; 2016.

30. ESRI. ArcGIS Desktop: Release 10.4. Redlands, CA: Environmental Systems Research Institute; 2016.

31. Distrito Federal (DF). Secretaria de Estado de Desenvolvimento Urbano e Habitação (SEDUH). Plano Diretor de Ordenamento Territorial Do Distrito Federal. Brasília: SEDUH; 2009.

32. Pesquisa Distrital por Amostra de Domicílios (PDAD). Pesquisa Distrital Por Amostra de Domicílios Do Distrito Federal. Brasília: PDAD; 2015.

33. Oliveira RM, Valla VV. As condições e as experiências de vida de grupos populares no Rio de Janeiro: repensando a mobilização popular no controle do dengue. Cad Saude Publica 2001; 17(Supl.):77-88.
34. Lagrotta MTF. Geoprocessamento de indicadores entomológicos na identificação de áreas, imóveis e recipientes "chaves" no controle do Aedes aegypti. Rio de Janeiro: Fiocruz; 2006.

35. Distrito Federal (DF). Secretaria de Estado de Saúde (SES). Dengue No DF Informe Epidemiológico; 2009. [acessado 2018 Maio 30]. Disponível em: http:// www.saude.df.gov.br/wp-conteudo/uploads/2018/08/ Informe_n_1_sobre_situacao_de_dengue_no_DF__10-02-09.pdf.

36. Distrito Federal (DF). Informe Epidemiológico de Dengue, Chikungunya e Zika No 52. Semana Epidemiológica 51 de 2016.; 2016. [acessado 2018 Maio 30]. Disponível em: http://www.saude.df.gov.br/wp-conteudo/ uploads/2018/08/Informativo_n_52_2016.pdf

37. Moura AM. A organização social do território e formas de provisão de moradia. In: Ribeiro R, Tenório G, Holanda F, organizadores. Brasília: Transformações $\mathrm{Na}$ Ordem Urbana. Rio de Janeiro: Letra Capital; 2015. p. 201-230.

38. Medeiros VAS, Barros AP. A organização social do território e mobilidade urbana. In: Ribeiro R, Tenório G, Holanda F, organizadores. Brasília: Transformações Na Ordem Urbana. Rio de Janeiro: Letra Capital; 2015. p. 252-284.

39. Barcellos C, Kreutz Pustai A, Weber MA, Varnieri Brito MR. Identificação de locais com potencial de transmissão de dengue em Porto Alegre através de técnicas de geoprocessamento. Rev Soc Bras Med Trop 2005; 38(3):246-250

40. Costa EB, Silveira B, Severo D, Araújo E, Beserra F, Carmo T. Metropolização, patrimonialização e potenciais de conflitos socioterritoriais em Brasília (DF). Espaço e Geogr. 2013; 16(1):325-367.

41. Paviani A. A construção injusta do espaço urbano. In: Paviani A. A Conquista Da Cidade: Movimentos Populares Em Brasília. Brasília: UnB; 1991. p. 115-142.

42. Souza SMC. Expansão urbana, centralidade e constituição de subcentros no Distrito Federal [dissertação]. Brasília: UnB; 2010.

43. Santana LS. Difusão espacial das epidemias de febre Zika no município de Salvador - Bahia, 2015 - 2016: a dispersão dessa doença possui o mesmo padrão das epidemias de dengue e febre chikungunya? [tese]. Rio de Janeiro: Fiocruz; 2018.

Artigo apresentado em 30/05/2019

Aprovado em 07/08/2019

Versão final apresentada em 13/11/2019 